\title{
AUTONOMOUS SCOOPING OF A ROCK PILE BY A WHEEL LOADER USING DISTURBANCE OBSERVER
}

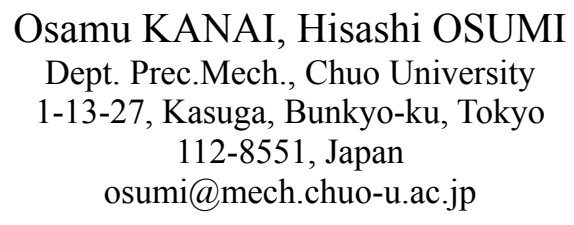

\author{
Shigeru SARATA \\ Intelligent Systems Institute, AIST \\ Namiki 1-2-1, Tsukuba, Ibaraki, \\ 305-8564, JAPAN
}

\author{
Masamitsu KURISU \\ Tokyo Denki University \\ Kanda-Nishiki-cho, Chiyoda-ku, \\ Tokyo 101-8457, Japan
}

\begin{abstract}
A controller for a miniature wheel loader is developed to scoop rock piles autonomously. During a scooping task operation, the load of the bucket varies momentarily according to the phase of scooping. Before the insertion of the bucket into the rock pile, the load is just the bucket weight, but after the insertion the reaction force from the rock piles is applied to the bucket. The values of the reaction forces changes significantly and they can not be identified in advance. To achieve autonomous loading, it is required for the bucket controller to work stably in both cases with the same algorithm. Therefore, a disturbance observer mechanism is installed into a miniature wheel loader "Yamazumi 3" to adapt various amounts of loads. Its effectiveness is verified by fundamental experiments.
\end{abstract}

Keywords: wheel loader, construction robot, autonomous scooping, disturbance observer

\section{Introduction}

Many kinds of construction machines have been automatized in recent years to promote unmanned construction. In particular, the automatic control of a wheel loader is strongly desired because of its versatility. However, it is much more difficult to automatize wheel loaders in comparison with the automatization of a damp truck because of the complexity of the soil or rock pile models. There are few studies dealing with the automatization of wheel loaders [1]. To automatize the wheel loaders the following three technical problems should be solved: 1) Obtaining rock pile models suitable for designing control algorithms of wheel loaders[2]-[5], 2) Generating bucket trajectory for scooping soils efficiently[6]-[10], and 3) Development of controllers which is applicable to the environment with rapidly changing external forces. The former two problems have been dealt with in many previous studies. Authors have also modeled the reaction forces from rock piles and also proposed a way to scoop rocks efficiently [10]. However, the last problem has not been dealt with up to now. Therefore, the third problem on the controllers is solved by installing disturbance observers to the loader controllers in this paper. Its effectiveness is verified by some fundamental experiments by using a miniature wheel loader "Yamazumi 3"[11].

In Section 2, the developed miniature wheel loader "Yamazumi 3" is introduced. In Section 3, the basic control algorithm is explained and the installed disturbance observer is shown. In Section 4, some fundamental experiments are done to verify the effectiveness of the developed controller.

\section{Abstract of Miniature Wheel Loader "Yamazumi"}

The developed miniature wheel loader is shown in Fig.1. This is a $1 / 10$ scale model of a practical one and named "Yamazumi". The body size is about $0.77[\mathrm{~m}] \mathrm{x} 0.26[\mathrm{~m}] \mathrm{x} 0.25[\mathrm{~m}]$ and its weight is about $22[\mathrm{~kg}]$. The radius of each wheel is $12[\mathrm{~cm}]$. The width of the bucket is about $25[\mathrm{~cm}]$ and it can scoop about $2[\mathrm{~kg}]$ rocks.

The structure of the loader is shown in Fig.2. The bucket is driven by two DC servo motors through a parallel link mechanism. The four wheels are driven by one motor through an universal joint and two differential gears. An articulated steering mechanism is used. The angles of all motors are measured by encoders, and torques are measured through their currents. A CPU board PCISA-3716EV-R3 (Pentium 3, 1.4GHz, OS:RTLinux) made by IEI is installed into the loader body as a controller. All motors are controlled by voltages sent from 10 bit DA converters on the interface board RIF-171-1 made by Ritech. The control sampling is $5[\mathrm{~ms}]$.

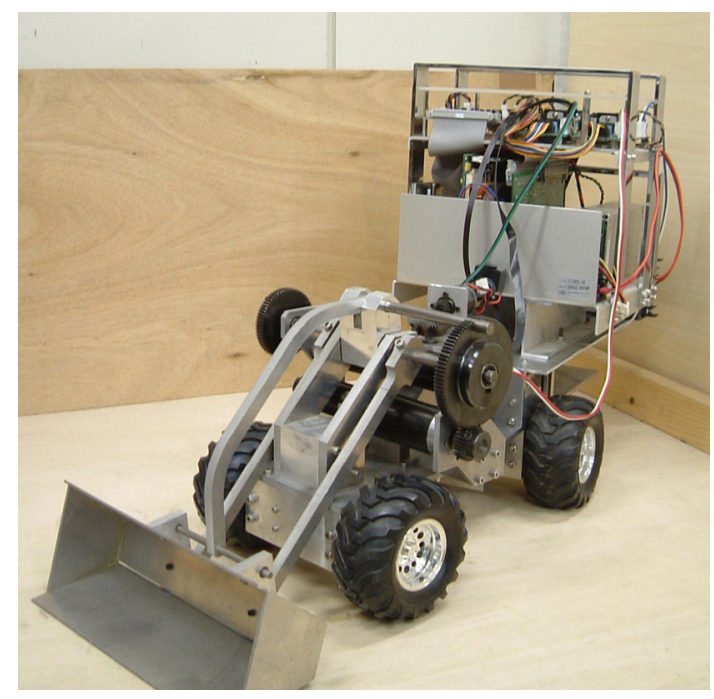

Fig.1 Miniature wheel loader "Yamazumi 3" 


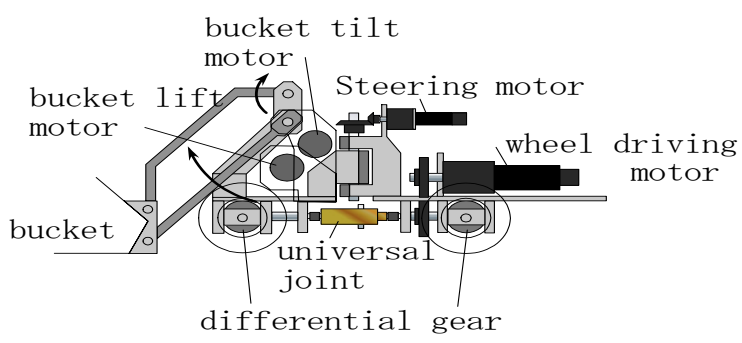

Fig.2 Miniature wheel loader "Yamazumi 3"

\section{Controller for "Yamazumi 3"}

\subsection{Basic servo control system}

The dynamics of the loader is neglected here because the motion of the loader is not so fast and the dominant term of the motor output is a compensation for the reaction forces from rock piles or gravity of rocks in the bucket.

The block diagram of the basic servo system is shown in Fig.3. First, a velocity feedback loop is added and the gain $k_{\mathrm{v}}$ is determined as large as possible with keeping the stability of the system. Then, a position feedback is added. The position gain $k_{\mathrm{p}}$ is also determined in the same manner as $k_{\mathrm{v}}$. This is a normal high gain servo system. Eq.(1).

The commanded torque to each motor is expressed as

$$
\tau_{d}=k_{v} k_{p}\left(\theta_{d}-\theta\right)-k_{v} \dot{\theta}
$$

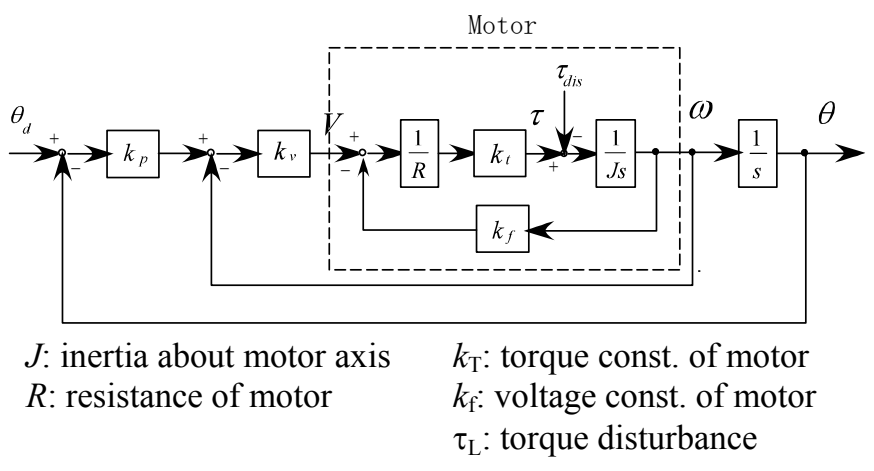

Fig.3 Block diagram of basic servo system for each motor

\subsection{Disturbance observer}

The designed servo system works well in free space motion, but once the bucket is inserted into a rock pile, a large external force is applied to the bucket. So, even if the feedback gain is designed as large as possible, the positioning error of the bucket inevitably appears during the scooping. If the bucket is filled with rocks, its positioning error may cause the collision of the bucket with a truck box.

The disturbance observer shown in Fig. 4 is installed into the servo system in Fig.3.

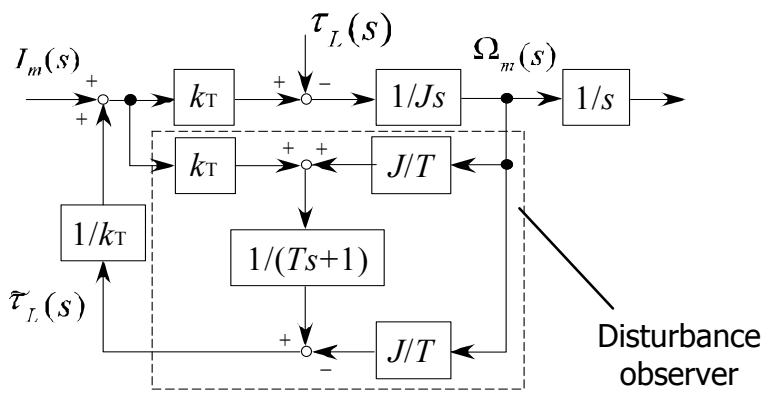

Fig.4 Block diagram of disturbance observer

In Fig.4, $J$ denotes the inertia of each motor and $T$ is a time constant of a low pass filter to cut sensor noises. $J$ changes according to the pose of the bucket, but the range of varying value is small because the angle ranges of the joints are also small. Therefore, $J$ is regarded as a constant. Time constant $T$ is determined by trial and error.

The disturbance torques is then estimated as Eq.(2) by the disturbance observer.

$$
\widetilde{\tau}_{L}(s)=\frac{1}{T s+1}\left(K_{T} I_{m}(s)+\frac{J}{T} \Omega_{m}(s)\right)-\frac{J}{T} \Omega_{m}(s)
$$

This estimated torque is added to the commanded torque calculated from Eq.(1). This disturbance observer is installed into all the joints.

\section{Experiments}

\subsection{Verification of effectiveness of disturbance observer}

A fundamental experiment is done to verify the effectiveness of the designed disturbance observer. In the experiment, the bucket is lifted up from $-48[\mathrm{deg}]$ to +48 [deg] without changing its pose as shown in Fig.5. During the motion, a $2[\mathrm{~kg}]$ weight is put on the bucket at the moment the bucket becomes horizontal.

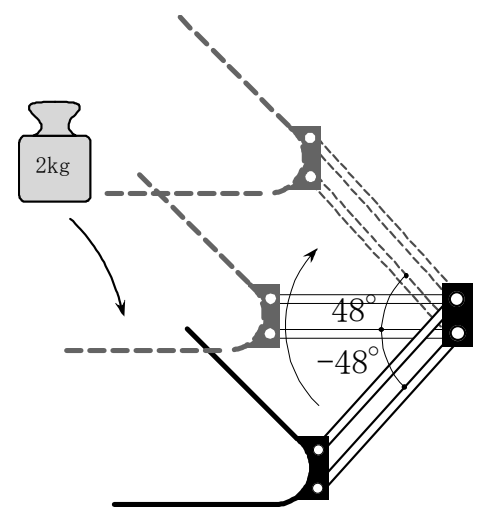

Fig. 5 Experiment for verification of the effectiveness of disturbance observer

The parameters of the system are listed in Table 1. The cut off frequency of the low pass filter is about $20 \mathrm{~Hz}$. The result is shown in Fig.6. The close up of the chart at the moment the weight is put and that at the moment the 
arm reaches the target angle are shown in Fig.7.

In Fig.7(a), the trajectory of the arm without disturbance observer deviates from the desired trajectory at the moment the weight is put, but the deviation with disturbance observer is not observed because the deviation is compensated in a few sampling times. In Fig.7(b), steady-state error is observed in the chart without disturbance observer, but it is completely compensated with disturbance observer.

Fig. 8 indicates the output of the disturbance observer. The estimated values changes to the curve expressing $2 \mathrm{~kg}$ load when the bucket angle becomes zero.

Table 1 Parameters of servo system

\begin{tabular}{|c|c|c|}
\hline \multicolumn{2}{|c|}{$k_{\mathrm{T}}[\mathrm{Nm}]$} & 0.0525 \\
\hline \multicolumn{2}{|c|}{$R[\Omega]$} & 2.05 \\
\hline \multicolumn{2}{|c|}{$k_{\mathrm{f}}[\mathrm{V} / \mathrm{rad} / \mathrm{s}]$} & 0.0525 \\
\hline \multicolumn{2}{|c|}{$J\left[\mathrm{kgm}^{2}\right]$} & 0.1466 \\
\hline \multicolumn{2}{|c|}{$T[\mathrm{~s}]$} & 0.008 \\
\hline \multirow{2}{*}{ gain } & $k_{\mathrm{V}}$ & 28 \\
\hline & $k_{\mathrm{P}}$ & 12 \\
\hline \multicolumn{2}{|c|}{ Gear reduction ratio } & 108:1 \\
\hline \multicolumn{2}{|c|}{ Sampling time [ms] } & 5 \\
\hline
\end{tabular}

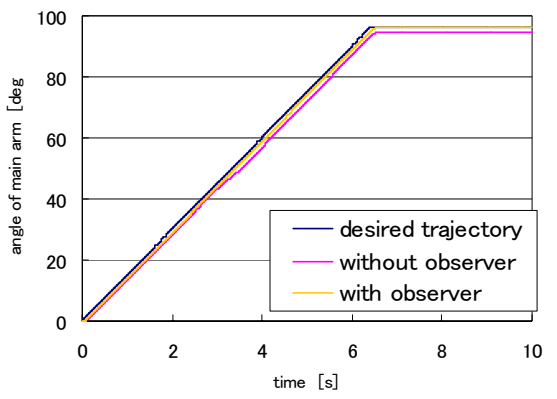

Fig.6 Experimental results of arm angles

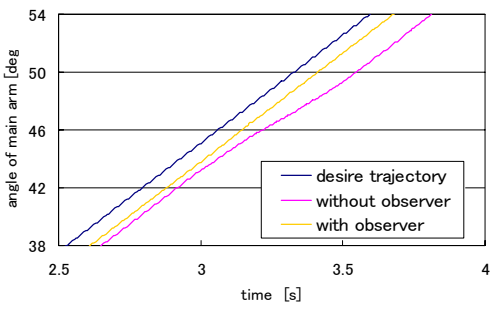

(a) Trajectory deviation

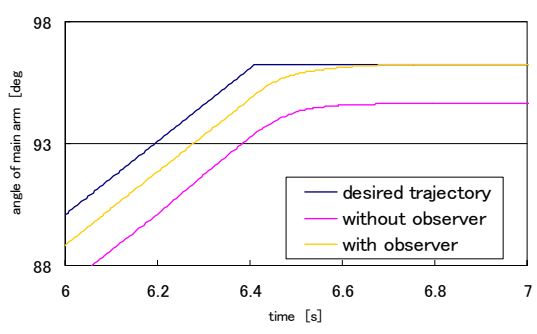

(b) Steady-state offset

Fig. 7 Close up of arm angles

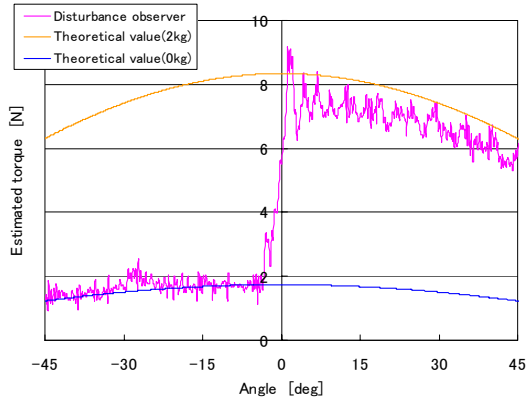

Fig.8 Output of disturbance observer

From these results, the effectiveness of the disturbance observer is verified.

\subsection{Scooping of rocks}

Next, scooping of rocks by the miniature wheel loader is carried out. The rock is crushed granite and the radius of each piece is about $4 \mathrm{~mm}$. Its specific gravity is about $2.7\left[\mathrm{~g} / \mathrm{cm}^{3}\right]$. The steps of scooping are designed in advance as follows:

Step1: The bucket is inserted into a rock pile in horizontal direction without tilting until the reaction force reaches a threshold value.(Fig.9(a))

Step2: After the reaction force becomes larger than the threshold, the bucket is lifted up. If the reaction force does not decrease, the bucket is simultaneously tilted.(Fig.9(b))

Step3: After the height of the bucket reaches a threshold, the bucket orientation is controlled straight up to avoid spilling rocks.(Fig.9(c))

As a result of the above scooping experiments, the bucket was always filled with about $2[\mathrm{~kg}]$ rocks, which shows the effectiveness of the proposed control law.

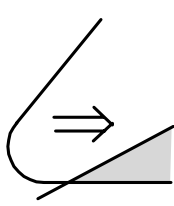

(a) step1

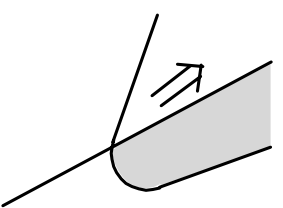

(b) step2

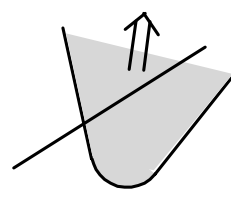

(c) step3
Fig.9 Scooping steps

\subsection{Bucket trajectory in scooping}

It was reported in a previous research[9] that the trajectory of the bucket generated by the same algorithm as step1-step3 becomes parallel to the slope of pile in scooping. This result is also examined by experiments here.

First, the slope angle of the pile is set as the repose angle 40 [deg]. Three threshold values of the step1, 30[N], $40[\mathrm{~N}]$ and $50[\mathrm{~N}]$ are used and the bucket trajectories are measured. The results are shown in Fig. 10.

From the chart, all the motion directions at the beginning of Step2 are the same as the repose angle, which corresponds with the previous report. 


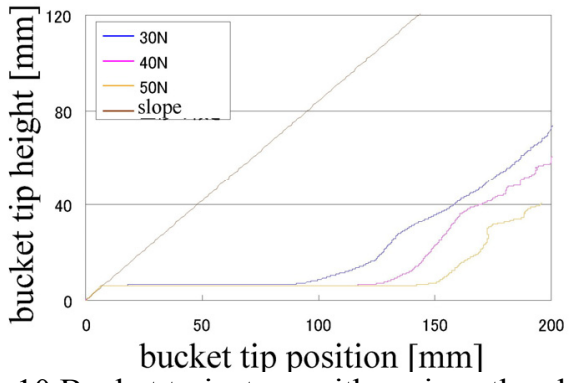

Fig.10 Bucket trajectory with various thresholds

Next, three angles of the slope are set and the bucket is controlled in the same manner. The threshold of the step 1 is $50 \mathrm{~N}$. The results are shown in Fig. 11.

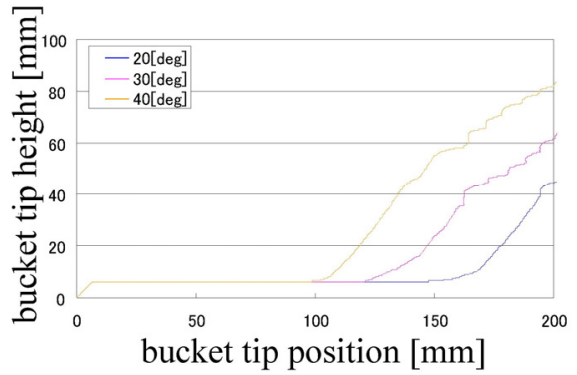

Fig.11 Bucket trajectory with various slope angles

From the chart, the motion directions at the beginning of Step2 are also the same as the repose angle although the slope angles are different. In many experiments, the repose angle is often used as a slope angle and that may be the reason why it was reported that the bucket moves parallel to the slope.

\section{Conclusions}

Servo control system with disturbance observer is developed for scooping rock piles by wheel loaders. First, a velocity feedback system with a large gain is designed, and a position feedback is added. A disturbance observer is also installed into the designed servo system in order to keep the commanded trajectory under large external disturbance forces.

The developed servo system was validated by some experiments using a miniature wheel loader "Yamazumi 3". The experimental results show the effectiveness of the developed control system.

Future works are the modeling of rocks suitable for designing scooping control algorithm and to apply the proposed control law to hydraulic actuator systems of the popular construction machines.

\section{Reference}

[1] Takashi Tsubouchi, Shigeru Sarata, Hisashi Osumi, Masamitsu Kurisu, Introduction of Yamazumi Project Trial for Autonomous Heavy Vehicles at Construction Site, Proc. of 2002 IEEE International Conf. on Industrial Technology, pp. 514-518, 2002.

[2] A. Hemami, "Force analysis in the scooping/loading operation of an LHD Loader" , Proc . mine Mechanization and Automation, Almgren, Kumar \& vaganas(eds), pp415-424, 1993.

[3] H.Takahashi et al.: Analysis on the Resistive Forces Acting on the Bucket of a Load-Haul-Dump Machine and a Wheel Loader in the Scooping Task, Advanced Robotics, Vol.13, No.2, pp.97-114, 1999.

[4] Don Carter, Andrew Alleyne, Load Modeling and Emulation for an Earthmoving Vehicle Powertrain, Proc. American Control Conference, pp.4963-4968, 2003.

[5] Choo Par Tan, Yahya H. Zweiri, Kaspar Althoefer, Lakmal D.Seneviratne; "Online Soil Parameter Estimation Scheme Based on Newton-Raphson Method for Autonomous Excavation", IEEE/ASME Trans. On Mechatronics, Vol.10, No.2, pp.221-229, 2005.

[6] A.Hemami; "Motion Trajectory Study in the Scooping Operation of an LHD-Loader", IEEE , 1994

[7] Sanjiv Singh, Howard Cannon :'Multi-Resolution Planning for Earthmoving", Proc. IEEE Int. Conf. Robotics and Automation, pp.121-126, 1998.

[8] Shigeru SARATA, Hisashi OSUMI, Yusuke HIRAI, Gen MATSUSHIMA and Takashi TSUBOUCHI, "Motion Control for Bucket of Wheel Loader Based on Shape of Pile and Resistance Force", Proc. of 18th Int. Symp. on Automation and Robotics in Construction, pp. 259-264, 2001.

[9] H.ZHANG, K.SATO and K.ITAKURA, "Strategy, Trajectory Generation and Control for $\operatorname{Scooping}\left(2^{\text {nd }}\right.$ report)", Journal of The Mining and Materials Processing Institute of Japan, Vol.117, p.947-954, 2001.(in Japanese)

[10] Shigeru Sarata, Hisashi Osumi, Yusuke Hirai, Gen Matsushima, "Trajectory Arrangement of Bucket Motion of Wheel Loader", Proc. of 20th International Symposium on Automation and Robotics in Construction, pp. 135-140, 2003.

[11] H.Osumi, S.Sarata, Y.Hirai, O.Kanai: "Analysis of reaction force with a scooping soil", Proc. Symp. on Construction Robot, pp201-208, 2004.(in Japanese) 